\section{PWE-167 ANVIL SUTURE PULL-THROUGH TECHNIQUE FOR LAPAROSCOPIC CIRCULAR STAPLED OESOPHAGOJEJUNOSTOMY: TECHNICAL REPORT AND OUTCOMES}

doi:10.1136/gutjnl-2012-302514d.167

${ }^{1} \mathrm{G}$ Bouras, ${ }^{*} \mathrm{~S}-\mathrm{W}$ Lee, ${ }^{2} \mathrm{E}$ Nomura, ${ }^{2} \mathrm{~T}$ Tokuhara, ${ }^{2} \mathrm{~N}$ Tanigawa. ${ }^{1}$ Upper Gl Surgery, Birmingham Heartlands Hospital, Birmingham, UK; ${ }^{2}$ Department of Gastrointestinal Surgery, Osaka Medical College, Takatsuki, Japan

Introduction The development of laparoscopic gastrectomy has lead to the need for intracorporeal stapled oesophagojejunostomy. Described techniques include overlap with a linear stapler ${ }^{1}$ and oral insertion of the anvil of a circular stapler. ${ }^{2}$ At our unit, we use a novel technique for laparoscopic oesophagojejunostomy by laparoscopic insertion of anvil into the gastrointestinal tract. Here we present our outcomes from laparoscopic oesophagojejunostomy performed by the anvil suture pull-through technique.

Methods After attaching a suture to the end of the spike of the anvil of a circular stapler, the anvil is inserted into the gastrointestinal tract through a proximal gastrotomy and pushed into the distal oesophagus. The oesophagus is divided below the anvil with a linear stapler. The suture poking out of the staple line is pulled and a small incision is made to allow the spike of the anvil to be delivered. The body of the circular stapler is inserted into the jejunum and combined with the anvil before firing the stapler and completing the double-stapled anastomosis. Intracorporeal oesophagojejunostomy using this technique was performed during laparoscopic proximal and total gastrectomy between 1998 and 2011.

Results A total of 82 anastomoses were performed using the anvil suture pull-through technique as part of 35 total and 47 proximal gastric resections for gastric cancer. 69 patients (84\%) had early gastric cancer. Anastomotic leakage occurred in three patients $(3.7 \%)$ and stenosis occurred in seven patients (8.5\%). Post-operative stasis occurred in one patient $(1.2 \%)$. No anastomotic bleeding was encountered. Recurrence at the site of the anastomosis was not detected during routine follow-up endoscopy in any of the patients.

Conclusion Intracorporeal oesophagojejunostomy using our anvil suture pull-through technique is feasible, safe and associated with good outcomes in the context of early gastric cancer. It is a reliable first-line technique and a useful alternative strategy when overlap is not possible or oral anvil insertion is contraindicated.

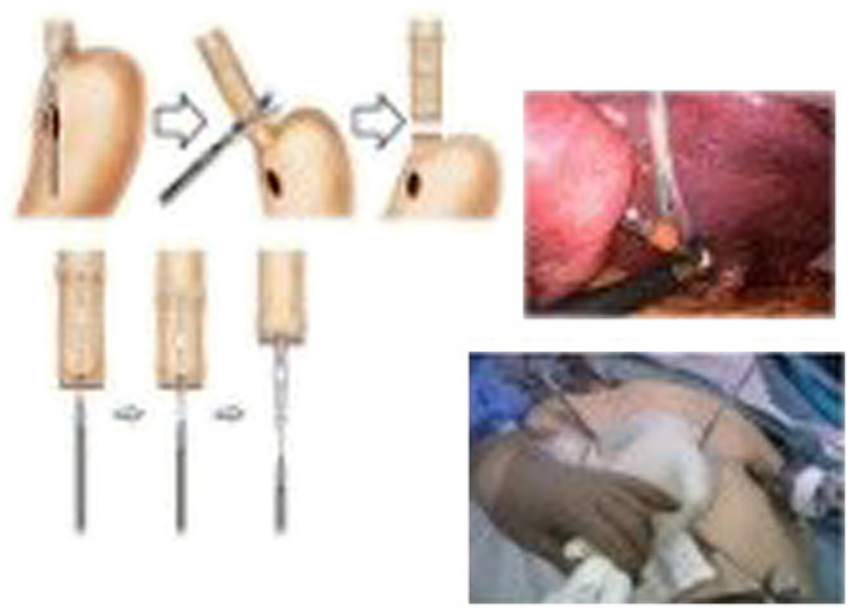

Abstract PWE-167 Figure 1

\section{REFERENCES}

1. Okabe H, Obama K, Tanaka E, et al. Intracorporeal esophagojejunal anastomosis after laparoscopic total gastrectomy for patients with gastric cancer. Surg Endosc 2009;23:2167-71

2. Jeong 0, Park YK. Intracorporeal circular stapling esophagojejunostomy using the transorally inserted anvil (OrVil) after laparoscopic total gastrectomy. Surg Endosc 2009;23:2624-30

\section{PWE-168 PALLIATIVE RESECTION FOR ADVANCED GASTRIC AND JUNCTIONAL ADENOCARCINOMA (AGJA): WHICH PATIENTS WILL BENEFIT FROM SURGERY?}

doi:10.1136/gutjnl-2012-302514d.168

${ }^{1} \mathrm{G}$ Piessen, ${ }^{*}{ }^{1} \mathrm{~W}$ B Robb, ${ }^{1} \mathrm{M}$ Messager, ${ }^{2} \mathrm{~J}$ H Lefevre, ${ }^{3} \mathrm{~V}$ Pichot-Delhaye, ${ }^{4} \mathrm{~A}$ Souadka, ${ }^{1} \mathrm{C}$ Mariette. ${ }^{1}$ Department of Digestive Surgery, Lille University Hospital, Lille, France; ${ }^{2}$ Department of Digestive Surgery, Sainte Antoine University Hospital, Paris, France; ${ }^{3}$ Department of Digestive Surgery, Lyon University Hospital, Lyon, France; ${ }^{4}$ Department of Digestive Surgery, Gustave Roussy Institute, Villejuif, France

Introduction Whereas palliative chemotherapy offers median survival of 10 months in AGJA, the survival impact of associated primary tumour resection is controversial. The objective of this study was to identify which patients with AGJA will benefit from palliative resection.

Methods Among 3202 patients with GJA registered in 19 French centres between January 1997 and January 2010 and scheduled for surgery, 677 benefited from palliative tumoural resection. Patients' characteristics were compared between palliative and curative groups. In the palliative group, prognostic factors were identified and the impact of each combination of these factors on survival was studied.

Results Median survival of AGJA patients resected with a palliative intent $(n=677)$ was longer than in non-resected patients $(n=532)$ (12.9 vs 8.5 months, $\mathrm{p}<0.001)$. Among resected patients, surgery was defined as palliative due to metastasis $(n=150,5.6 \%)$, localised $(n=122,4.6 \%)$ or diffuse $(n=62,2.3 \%)$ peritoneal carcinomatosis (PC), or incomplete tumoural resection ( $n=343,12.8 \%)$. Overall median survival was 30.0 months, significantly shorter after palliative than curative resection ( 11.9 vs 48.2 months, $p<0.001$ ) Predictors of postoperative mortality were ASA score III-IV $(p<0.001)$ and palliative resection $(p=0.020)$, justifying palliative resection only in ASA I-II patients. Independent prognostic factors in the palliative group were solid organ metastasis $(p=0.009)$, localised PC $(p=0.004)$, diffuse PC $(p=0.046)$ and signet ring cell histology (SRC) $(p=0.020)$. In ASA I-II patients, patients with diffuse PC, metastasis combined with PC or localised PC of SRC had median survivals from 1.3 to 9.3 months. Patients with incomplete resection without metastasis or PC, organ metastasis without PC, or localised PC without SRC had median survival from 12.0 to 18.3 months.

Conclusion In AGJA, only ASA I-II patients, presenting with limited tumoural extension will benefit from palliative resection in combination with chemotherapy. Other clinical presentations have to be enrolled in exclusive palliative chemotherapy programs. Clinical trial registration number Clinical Trial.gov identifier NCT01249859.

Competing interests None declared.

\section{PWE-169 ANALYSIS OF FAECAL VOLATILES FROM YOUNG CHILDREN INFECTED WITH AND WITHOUT ROTAVIRUS}

doi:10.1136/gutjnl-2012-302514d.169

${ }^{1} \mathrm{H}$ Al-Kateb, ${ }^{*} \mathrm{~N}$ Cunliffe, ${ }^{1} \mathrm{~B}$ deLacy Costello, ${ }^{2} \mathrm{C}$ Probert, ${ }^{1} \mathrm{~N}$ Ratcliffe. ${ }^{1}$ Applied sciences, University of West of England/Centre of Research in Biosciences, 